\title{
Application of the Fast Expansion Method in Space-Related Problems
}

\author{
Mikhail Ivanovich Popov ${ }^{1}$, Aleksey Vasilyevich Skrypnikov ${ }^{1}$, Vyacheslav Gennadievich Kozlov ${ }^{2, *}$, \\ Alexey Viktorovich Chernyshov ${ }^{2}$, Alexander Danilovich Chernyshov ${ }^{1}$, Sergey Yurievich Sablin ${ }^{1}$, \\ Vladimir Valentinovich Nikitin ${ }^{1}$, Roman Alexandrovich Druzhinin ${ }^{2}$ \\ ${ }^{1}$ Voronezh State University of Engineering Technologies, Voronezh, Russian Federation \\ ${ }^{2}$ Voronezh State Agrarian University named after Emperor Peter the Great, Voronezh, Russian Federation
}

Received October 27, 2021; Revised January 26, 2022; Accepted February 21, 2022

\section{Cite This Paper in the following Citation Styles}

(a): [1] Mikhail Ivanovich Popov, Aleksey Vasilyevich Skrypnikov, Vyacheslav Gennadievich Kozlov, Alexey Viktorovich Chernyshov, Alexander Danilovich Chernyshov, Sergey Yurievich Sablin, Vladimir Valentinovich Nikitin, Roman Alexandrovich Druzhinin , "Application of the Fast Expansion Method in Space-Related Problems," Mathematics and Statistics, Vol. 10, No. 2, pp. 320 - 328, 2022. DOI: 10.13189/ms.2022.100206.

(b): Mikhail Ivanovich Popov, Aleksey Vasilyevich Skrypnikov, Vyacheslav Gennadievich Kozlov, Alexey Viktorovich Chernyshov, Alexander Danilovich Chernyshov, Sergey Yurievich Sablin, Vladimir Valentinovich Nikitin, Roman Alexandrovich Druzhinin (2022). Application of the Fast Expansion Method in Space-Related Problems. Mathematics and Statistics, 10(2), 320 - 328. DOI: 10.13189/ms.2022.100206.

Copyright $\bigcirc 2022$ by authors, all rights reserved. Authors agree that this article remains permanently open access under the terms of the Creative Commons Attribution License 4.0 International License

\begin{abstract}
In the paper, numerical and approximate analytical solutions for the problem of the motion of a spacecraft from a starting point to a final point during a certain time are obtained. The unpowered and powered portions of the flight are considered. For a numerical solution, a finite-difference scheme of the second order of accuracy is constructed. The space-related problem considered in the study is essentially nonlinear, which necessitates the use of trigonometric interpolation methods to replace the task of calculating the Fourier coefficients with the integral formulas by solving the interpolation system. One of the simplest options for trigonometric sine interpolation on a semi-closed segment $[-a, a)$, where the right end is not included in the general system of interpolation points, is considered. In order to maintain the conditions of orthogonality of sines, an even number of $2 \mathrm{M}$ calculation points is uniformly applied to the segment. The sine interpolation theorem is proved and a compact formula is given for calculating the interpolation coefficients. A general theory of fast sine expansion is given. It is shown that in this case, the Fourier coefficients decrease much faster with the increase in serial number compared to the Fourier coefficients in the classical case. This property allows reducing the number of terms taken into account in the Fourier series, as well as the amount of
\end{abstract}

computer calculations, and increasing the accuracy of calculations. The analysis of the obtained solutions is carried out, and their comparison with the exact solution of the test problem is proposed. With the same calculation error, the time spent on a computer using the fast expansion method is hundreds of times less than the time spent on classical finite-difference method.

Keywords Spacecraft, Fast Expansion Method, Finite-Difference Scheme

\section{Introduction}

The calculation of the spacecraft trajectory in the atmosphere of the earth or other planets is of great importance in the preparation of flights of satellites and space vehicles [1]. First of all, it is necessary to assess the possibility of launching a payload using a launch vehicle into a given orbit. It is equally important to obtain initial information on flight trajectory parameters for calculating onboard flight mission coefficients. Such a calculation is needed to implement optimal control to minimize costs. Modernity dictates new tasks for the development of areas 
of outer space remote from the Earth. The cost of such flights is very high, so the task of calculating the trajectory becomes more relevant [2].

In many practical spacecraft guidance, navigation, and control systems, the trajectory planning phase often needs to take into account many performance metrics and various types of uncertainties. To solve this problem, methods of multicriteria optimization, as well as algorithms for stochastic optimization of the spacecraft trajectory, are used [3].

The mathematical model of the spacecraft motion on the powered portion of the trajectory is a system of nonlinear differential equations. The integration of such a system presents significant difficulties since the result of integration cannot be reduced to an analytical form [4]. At present, analytical [5-7] and semi-analytical [8] methods for solving such systems are known. Nevertheless, boundary value problems with essential nonlinearity for both ordinary differential equations and partial differential equations are solved using numerical methods [9-11] and their modifications [12].

The calculation of a spacecraft's flight path requires high accuracy. For its implementation on a sufficiently large time interval, it is necessary to consider, firstly, many significant figures, and secondly, a large number of nodes in the computational grid, which requires significant memory consumption and an increase in computation time. In this regard, methods are used to accelerate the convergence of different schemes [13]. In recent times, и spectral and pseudospectral [14] methods have also been used.

In the present paper, an approximate analytical method is considered, the fast expansion method [15-17]. This method has high accuracy and does not require significant computer resources.

\section{Formulation of the Problem}

A spaceship is a complex system of material points for which the theorem on the motion of the center of mass is true. For this reason, in the well-known studies [5, 7], the motion of a spacecraft is reduced to the analysis of the motion of its center of mass. In the considered simplified model, the issues of rotation of the spacecraft are not touched upon. Let us write the equations of the spacecraft motion in the form of a system of differential equations in the geocentric equatorial rotating coordinate system:

$$
\left\{\begin{array}{l}
\ddot{x}+\beta \dot{x}+\alpha x /\left(x^{2}+y^{2}+z^{2}\right)^{3 / 2}=P_{x}, \\
\ddot{y}+\beta \dot{y}+\alpha y /\left(x^{2}+y^{2}+z^{2}\right)^{3 / 2}=P_{y}, \\
\ddot{z}+\beta \dot{z}+\alpha z /\left(x^{2}+y^{2}+z^{2}\right)^{3 / 2}=P_{z} .
\end{array}\right.
$$

Here, $R_{E}$ is the Earth's radius, $\alpha=g R_{E}^{2}$ is the coefficient of attraction to the Earth of a unit mass, $g$ is the acceleration of gravity, $\beta$ is the aerodynamic drag coefficient, $P_{x}, P_{y}, P_{z}$ are the components of the reactive force.

As a test example, let us choose the functions $P_{x}, P_{y}, P_{z}$, so that there is an exact solution to the problem: $x^{*}=a \cos \omega t, y^{*}=a \sin \omega t, z^{*}=R_{3}+w t$. Then,

$$
\begin{aligned}
& P_{x}=-a \omega^{2} \cos \omega t-\beta a \omega \sin \omega t+\alpha a \cos \omega t /\left((a \cos \omega t)^{2}+(a \sin \omega t)^{2}+\left(R_{3}+w t\right)^{2}\right)^{(3 / 2)}, \\
& P_{y}=-a \omega^{2} \sin \omega t+\beta a \omega \cos \omega t+\alpha a \sin \omega t /\left((a \cos \omega t)^{2}+(a \sin \omega t)^{2}+\left(R_{3}+w t\right)^{2}\right)^{(3 / 2)}, \\
& P_{z}=\beta w+\alpha\left(R_{3}+w t\right) /\left((a \cos \omega t)^{2}+(a \sin \omega t)^{2}+\left(R_{3}+w t\right)^{2}\right)^{(3 / 2)} .
\end{aligned}
$$

The boundary conditions take the form:

$$
\left\{\begin{array}{c}
x(0)=a, y(0)=0, z(0)=R_{3}, \\
x\left(t_{0}\right)=x_{t_{0}}, y\left(t_{0}\right)=y_{t_{0}}, z\left(t_{0}\right)=z_{t_{0}} .
\end{array}\right.
$$

In the problem (1), (2), the unknowns are the coordinates $x(t), y(t), z(t)$ of the center of mass of the spacecraft. The components of the reactive force are selected so that the ship moves along a helical line. Having the exact solution, one can calculate the absolute error of the ship location, its velocity and acceleration, which allows comparing the numerical and approximate analytical solutions.

The considered problem is a test problem to analyze the advantages of the fast expansion method compared to the classical finite-difference method. In this regard, aerodynamic drag is taken proportional to the first degree of velocity, since taking nonlinear terms into account does not create fundamental difficulties, just increasing the time of computational experiments [18].

\section{Fast Expansion Method}

In order to solve the problem (1), (2) by the fast expansion method, each unknown function $f(t)$ is represented by the sum of a special boundary function $M_{2 k}$ and the Fourier series on a given interval

$$
f(t)=M_{2 k}(t)+\sum_{m=1}^{N} f_{m} \sin m \pi t / t_{0} \quad, \quad t \in\left[0, t_{0}\right]
$$

The time $t_{0}$ is the time taken to move along the power motion. The boundary function $M_{2 k}, k=0,1,2, \ldots$ has the following construction. Let introduce the polynomials $P_{0}=1-t / t_{0}$ and $Q_{0}=t / t_{0}$. Let functions $P_{2 k}$ and $Q_{2 k}$ be recurrent to integral relations: $P_{2 k}=\int\left(\int P_{2(k-1)} d t\right) d t$, $Q_{2 k}=\int\left(\int Q_{2(k-1)} d t\right) d t$. Constant integrations are found from the boundary conditions $P_{2 k}(0)=P_{2 k}\left(t_{0}\right)=0$ and $Q_{2 k}(0)=Q_{2 k}\left(t_{0}\right)=0$. Let define the function $M_{0}=x(0) P_{0}+x\left(t_{0}\right) Q_{0}$. The boundary function $M_{2 k}$ is set by the recurrence relation:

$$
M_{2 k}=M_{2(k-1)}+x^{(2 k)}(0) P_{2 k}+x^{(2 k)}\left(t_{0}\right) Q_{2 k}
$$


In the case of Dirichlet boundary conditions, fast polynomials $P_{2 k}(x), Q_{2 k}(x)$ are calculated by the following recurrent integral formulas:

$$
\begin{gathered}
P_{0}(x)=1-x, Q_{0}(x)=x, P_{2 k}(x)=\int_{0}^{x}\left[\int_{0}^{t_{1}} P_{2 k-2}\left(t_{2}\right) d t_{2}\right] d t_{1}-x \int_{0}^{1}\left[\int_{0}^{t_{1}} P_{2 k-2}\left(t_{2}\right) d t_{2}\right] d t_{1}, \\
\left.Q_{2 k}(x)=\int_{0}^{x}\left[\int_{0}^{t_{1}} Q_{2 k-2}\left(t_{2}\right) d t_{2}\right] d t_{1}-x \int_{0}^{1} \int_{0}^{t_{1}} Q_{2 k-2}\left(t_{2}\right) d t_{2}\right] d t_{1}, \\
k=1 \div p, 0 \leq t_{2} \leq t_{1}, \quad 0 \leq t_{1} \leq x, 0 \leq x \leq t_{0}
\end{gathered}
$$

Polynomials $P_{0}(x), Q_{0}(x)$ are reproducing; through them, with the use of double integrals, all other polynomials with even indices are found. Fast polynomials $P_{2 k}(x), Q_{2 k}(x)$ can also be calculated from the solution of boundary-value differential problems with zero Dirichlet boundary conditions, for which they were created [17]:

$$
\begin{gathered}
P_{2 k}^{\prime \prime}(x)=P_{2 k-2}(x) \quad, \quad P_{2 k}(0)=P_{2 k}\left(t_{0}\right)=0, k \neq 0, k=1 \div p, \\
Q_{2 k}^{\prime \prime}(x)=Q_{2 k-2}(x) \quad, \quad Q_{2 k}(0)=Q_{2 k}\left(t_{0}\right)=0
\end{gathered}
$$

The index $2 k$ in (3) must be not lower than the order of the highest derivative in the differential equation, into which expansion (3) is substituted. The fast expansion in (3) allows term-by-term differentiation of the Fourier series $2 k$ times, while the series remain rapidly converging. Due to the special construction of polynomials $P_{2 k}(x), Q_{2 k}(x)$ in (4), the boundary function $M_{2 k}(x)$ significantly increases the rate of convergence of the Fourier series. With the increase in the $2 k$ order, the convergence rate substantially increases [19]. Polynomials $P_{2 m}(x), Q_{2 m}(x)$ with even indices are used in fast sine expansions.

For definiteness and simplicity of further calculations, let choose a boundary function in (3) of not high order at $k=1$, i.e. $M_{2}$ :

$$
\begin{aligned}
& x(t)=x(0)\left(1-t / t_{0}\right)+x\left(t_{0}\right) t / t_{0}+\ddot{x}(0)\left(t^{2} / 2-t^{3} / 6 t_{0}-t t_{0} / 3\right)+ \\
&+\ddot{x}\left(t_{0}\right)\left(t^{3} / 6 t_{0}-t t_{0} / 6\right)+\sum_{m=1}^{N} x_{m} \sin m \pi t / t_{0}, \\
& y(t)=y(0)\left(1-t / t_{0}\right)+y\left(t_{0}\right) t / t_{0}+\ddot{y}(0)\left(t^{2} / 2-t^{3} / 6 t_{0}-t t_{0} / 3\right)+ \\
&+\ddot{y}\left(t_{0}\right)\left(t^{3} / 6 t_{0}-t t_{0} / 6\right)+\sum_{m=1}^{N} y_{m} \sin m \pi t / t_{0}, \\
& z(t)=z(0)\left(1-t / t_{0}\right)+z\left(t_{0}\right) t / t_{0}+\ddot{z}(0)\left(t^{2} / 2-t^{3} / 6 t_{0}-t t_{0} / 3\right)+ \\
&+\ddot{z}\left(t_{0}\right)\left(t^{3} / 6 t_{0}-t t_{0} / 6\right)+\sum_{m=1}^{N} z_{m} \sin m \pi t / t_{0} .
\end{aligned}
$$

Let demonstrate a fast decrease in the Fourier coefficients $x_{m} \sim(m \pi)^{-4}$ on the example for $x(t)$. Let express the Fourier series from (6) for $x(t)$ :

$$
\begin{gathered}
\sum_{m=1}^{N} x_{m} \sin m \pi t / t_{0}=x(t)-x(0)\left(1-t / t_{0}\right)-x\left(t_{0}\right) t / t_{0}- \\
-\ddot{x}(0)\left(t^{2} / 2-t^{3} / 6 t_{0}-t t_{0} / 3\right)-\ddot{x}\left(t_{0}\right)\left(t^{3} / 6 t_{0}-t t_{0} / 6\right)
\end{gathered}
$$

Hence, for the coefficients $x_{m}$ we have the integral formula:

$$
x_{m}=\frac{2}{t_{0}} \int_{0}^{t_{0}}\left(\begin{array}{l}
x(t)-x(0)\left(1-t / t_{0}\right)-x\left(t_{0}\right) t / t_{0}- \\
-\ddot{x}(0)\left(t^{2} / 2-t^{3} / 6 t_{0}-t t_{0} / 3\right)-\ddot{x}\left(t_{0}\right)\left(t^{3} / 6 t_{0}-t t_{0} / 6\right)
\end{array}\right) \sin m \pi \frac{t}{t_{0}} d t
$$
(7):

$$
\begin{gathered}
x_{m}=-\frac{2}{m \pi} \int_{0}^{t_{0}}\left(\begin{array}{l}
x(t)-x(0)\left(1-t / t_{0}\right)-x\left(t_{0}\right) t / t_{0}- \\
-\ddot{x}(0)\left(t^{2} / 2-t^{3} / 6 t_{0}-t t_{0} / 3\right)-\ddot{x}\left(t_{0}\right)\left(t^{3} / 6 t_{0}-t t_{0} / 6\right)
\end{array}\right) d \cos m \pi \frac{t}{t_{0}}= \\
=\frac{2}{m \pi} \int_{0}^{t_{0}}\left(\begin{array}{l}
x^{\prime}(t)+x(0) / t_{0}-x\left(t_{0}\right) / t_{0}- \\
-\ddot{x}(0)\left(t-t^{2} / 2 t_{0}-t_{0} / 3\right)-\ddot{x}\left(t_{0}\right)\left(t^{2} / 2 t_{0}-t_{0} / 6\right)
\end{array}\right) \cos m \pi \frac{t}{t_{0}} d t= \\
=\frac{2 t_{0}}{(m \pi)^{2}} \int_{0}^{t_{0}}\left(\begin{array}{l}
x^{\prime}(t)+x(0) / t_{0}-x\left(t_{0}\right) / t_{0}- \\
-\ddot{x}(0)\left(t-t^{2} / 2 t_{0}-t_{0} / 3\right)-\ddot{x}\left(t_{0}\right)\left(t^{2} / 2 t_{0}-t_{0} / 6\right)
\end{array}\right) d \sin m \pi \frac{t}{t_{0}}= \\
=-\frac{2 t_{0}}{(m \pi)^{2}} \int_{0}^{t_{0}}\left(\ddot{x}(t)-\ddot{x}(0)\left(1-\frac{t}{t_{0}}\right)-\ddot{x}\left(t_{0}\right) \frac{t}{t_{0}}\right) \sin m \pi \frac{t}{t_{0}} d t= \\
=\frac{2 t_{0}^{2}}{(m \pi)^{3}} \int_{0}^{t_{0}}\left(\ddot{x}(t)-\ddot{x}(0)\left(1-\frac{t}{t_{0}}\right)-\ddot{x}\left(t_{0}\right) \frac{t}{t_{0}}\right) d \cos m \pi \frac{t}{t_{0}}= \\
=-\frac{2 t_{0}^{2}}{(m \pi)^{3}} \int_{0}^{t_{0}}\left(x^{\prime \prime \prime}(t)+\frac{\ddot{x}(0)-\ddot{x}\left(t_{0}\right)}{t_{0}}\right) \cos m \pi \frac{t}{t_{0}} d t= \\
=-\frac{2 t_{0}^{3}}{(m \pi)^{4}} \int_{0}^{t_{0}}\left(x^{\prime \prime \prime}(t)+\frac{\ddot{x}(0)-\ddot{x}\left(t_{0}\right)}{t_{0}}\right) d \sin m \pi \frac{t}{t_{0}}=\frac{2 t_{0}^{3}}{(m \pi)^{4}} \int_{0}^{t_{0}} x^{(4)}(t) \sin m \pi \frac{t}{t_{0}} d t
\end{gathered}
$$

Q.E.D. Similar estimates are obtained for $y_{m}$ and for $z_{m}$. Hence, the solution of the system in the form of (6) guarantees the possibility of its term-by-term double differentiation in time. When using boundary functions of higher order $2 k \geq 4$, the degree of decrease in the Fourier coefficients increases in proportion to the order of the boundary function and is equal to $2 k+2$.

The expressions for the coordinates of the flight path are substituted from (6) to the equation of motion (1):

$$
\begin{gathered}
\ddot{x}(0)\left(1-t / t_{0}\right)+\ddot{x}\left(t_{0}\right) t / t_{0}-\sum_{m=1}^{N} x_{m}\left(m \pi / t_{0}\right)^{2} \sin m \pi t / t_{0}+\alpha x /\left(x^{2}+y^{2}+z^{2}\right)^{3 / 2}+ \\
+\beta\left[\left(x\left(t_{0}\right)-x(0)\right) / t_{0}+\ddot{x}(0)\left(t-t^{2} /\left(2 t_{0}\right)-t_{0} / 3\right)+\ddot{x}\left(t_{0}\right)\left(t^{2} /\left(2 t_{0}\right)-t_{0} / 6\right)+\right. \\
\left.+\sum_{m=1}^{N} x_{m} m \pi / t_{0} \cos m \pi t / t_{0}\right]=P_{x}, \\
\ddot{y}(0)\left(1-t / t_{0}\right)+\ddot{y}\left(t_{0}\right) t / t_{0}-\sum_{m=1}^{N} y_{m}\left(m \pi / t_{0}\right)^{2} \sin m \pi t / t_{0}+\alpha y /\left(x^{2}+y^{2}+z^{2}\right)^{3 / 2}+ \\
+\beta\left[\left(y\left(t_{0}\right)-y(0)\right) / t_{0}+\ddot{y}(0)\left(t-t^{2} /\left(2 t_{0}\right)-t_{0} / 3\right)+\ddot{y}\left(t_{0}\right)\left(t^{2} /\left(2 t_{0}\right)-t_{0} / 6\right)+\right. \\
\left.+\sum_{m=1}^{N} y_{m} m \pi / t_{0} \cos m \pi t / t_{0}\right]=P_{y}, \\
\ddot{z}(0)\left(1-t / t_{0}\right)+\ddot{z}\left(t_{0}\right) t / t_{0}-\sum_{m=1}^{N} z_{m}\left(m \pi / t_{0}\right)^{2} \sin m \pi t / t_{0}+\alpha z /\left(x^{2}+y^{2}+z^{2}\right)^{3 / 2}+ \\
+\beta\left[\left(z\left(t_{0}\right)-z(0)\right) / t_{0}+\ddot{z}(0)\left(t-t^{2} /\left(2 t_{0}\right)-t_{0} / 3\right)+\ddot{z}\left(t_{0}\right)\left(t^{2} /\left(2 t_{0}\right)-t_{0} / 6\right)+\right. \\
\left.+\sum_{m=1}^{N} z_{m} m \pi / t_{0} \cos m \pi t / t_{0}\right]=P_{z},
\end{gathered}
$$

Hence, for the coefficients $x_{m}$ we have the intLet find unknown $x(0)$ and $x\left(t_{0}\right)$ from the boundary conditions (2). Thus, in (8) the constant values are unknown:

$$
\ddot{x}(0), \ddot{x}\left(t_{0}\right), x_{m}, \ddot{y}(0), \ddot{y}\left(t_{0}\right), y_{m}, \ddot{z}(0), \ddot{z}\left(t_{0}\right), z_{m}, m=1 \div N
$$

Due to the nonlinearity of the problem for finding unknowns (9), the pointwise method is used, which in the 
literature is also called the collocation method, or the method of trigonometric interpolation.

\section{The Method of Trigonometric Sine Interpolation}

Trigonometric interpolation with various basis functions in a Hilbert space will be considered for applied purposes. It is especially convenient to apply trigonometric sine interpolation when considering nonlinear boundary value problems. Let there exist some functions $f(x) \in L_{p}^{\alpha}([-a, a])$ whose values are known only at the points $x_{j}=j a / M, j=-M \div M$ of uniform division of the segment $x \in[0, a]$, where $L_{p}^{\alpha}-$ is the classes of Sobolev-Liouville spaces. Let represent $f(x)$ on a double segment $x \in[-a, a]$ as a fast sine expansion with the second-order boundary function $M_{2}(x)$ :

$$
\begin{gathered}
f(x)=M_{2}(x)+\psi(x), \\
M_{2}(x)=f(0)\left(1-\frac{x}{a}\right)+f(a) \frac{x}{a}+f^{\prime \prime}(0)\left(\frac{x^{2}}{2}-\frac{x^{3}}{6 a}-\frac{a x}{3}\right)+f^{\prime \prime}(a)\left(\frac{x^{3}}{6 a}-\frac{a x}{6}\right)
\end{gathered}
$$

We define the function $f(x)$ to the negative half $x \in[-a, 0]$ so that $\psi(x)$ is odd. Then from the oddness condition $\psi(x)=-\psi(-x)$ we get:

$$
\begin{gathered}
\psi(x)=f(x)-M_{2}(x), \psi(x)=-\psi(-x), \quad x \in[0, a] \Rightarrow \\
f(x)-\left[f(0)\left(1-\frac{x}{a}\right)+f(a) \frac{x}{a}+f^{\prime \prime}(0)\left(\frac{x^{2}}{2}-\frac{x^{3}}{6 a}-\frac{a x}{3}\right)+f^{\prime \prime}(a)\left(\frac{x^{3}}{6 a}-\frac{a x}{6}\right)\right]= \\
=-\left[f(-x)-\left[f(0)\left(1+\frac{x}{a}\right)-f(a) \frac{x}{a}+f^{\prime \prime}(0)\left(\frac{x^{2}}{2}+\frac{x^{3}}{6 a}+\frac{a x}{3}\right)-f^{\prime \prime}(a)\left(\frac{x^{3}}{6 a}-\frac{a x}{6}\right)\right]\right]
\end{gathered}
$$

Hence, the definition of $f(-x)$ is found on the negative half of the segment:

$$
f(-x)=-f(x)+2 f(0)+f^{\prime \prime}(0) x^{2} \quad, \quad x \in[0, a]
$$

Odd $\psi(x)$, defined by the difference $\psi(x)=f(x)-M_{2}(x)$ in (11), is represented by the Fourier sine series on a double segment $x \in[-a, a]$ :

$$
\psi(x)=\sum_{m=-M}^{M-1} \psi_{m} \sin m \pi \frac{x}{a}
$$

Here, the basic functions are $\sin \left(n \pi x_{j} / a\right)$ and in total the term is not used for $m=M$. In fact, here we will prove orthogonality $\sin \left(n \pi x_{j} / a\right)$ on the interval $[-a, a-a / M]$, i.e. without the last small segment on the right. Interpolation coefficients $\psi_{m}$ can be found explicitly from a closed algebraic system:

$$
\psi\left(x_{j}\right)=\sum_{m=-M}^{M-1} \psi_{m} \sin m \pi \frac{x_{j}}{a}, x_{j}=j \frac{a}{M} \quad, j=-M \div M-1
$$

The orthogonality of functions $\sin \left(n \pi x_{j} / a\right)$ is used. To do this, the left and right sides of (14) are multiplied by $\sin \left(n \pi x_{j} / a\right), n=-M \div M$, and added up by the index $j$ :

$$
\sum_{j=-M}^{M-1} \psi\left(x_{j}\right) \sin n \pi \frac{x_{j}}{a}=\sum_{m=-M}^{M=1} \psi_{m} \sum_{j=-M}^{M-1} \sin m \pi \frac{x_{j}}{a} \sin n \pi \frac{x_{j}}{a}, n=-M \div M-1
$$

Let demonstrate that for $m \neq n$ the second sum on the right-hand side of (15) is equal to zero:

$$
\sum_{j=-M}^{M-1} \sin \left(n \pi \frac{j}{M}\right) \sin \left(m \pi \frac{j}{M}\right)=0, \quad m \neq n
$$

Let rewrite (16) through cosines:

$$
\frac{1}{2} \sum_{j=-M}^{M-1}\left(\cos \left((m-n) \pi \frac{j}{M}\right)-\cos \left((m+n) \pi \frac{j}{M}\right)\right)=0, \quad m \neq n
$$

In (17) for cosines the complex Euler formula is used:

$$
\begin{gathered}
\sum_{j=-M}^{M-1}\left(\cos (m-n) \pi \frac{j}{M}-\cos (m+n) \pi \frac{j}{M}\right)= \\
=\frac{1}{2} \sum_{j=-M}^{M-1}\left(\exp \left(i(m-n) \pi \frac{j}{M}\right)+\exp \left(-i(m-n) \pi \frac{j}{M}\right)\right)- \\
-\frac{1}{2} \sum_{j=-M}^{M-1}\left(\exp \left(i(m+n) \pi \frac{j}{M}\right)+\exp \left(-i(m+n) \pi \frac{j}{M}\right)\right)
\end{gathered}
$$

Let denote $q_{ \pm}=\exp (i(m \pm n) \pi / M)$ and represent (18) by the expression:

$$
\begin{gathered}
\sum_{j=-M}^{M-1}\left(\exp \left(i(m-n) \pi \frac{j}{M}\right)+\exp \left(-i(m-n) \pi \frac{j}{M}\right)\right)- \\
-\sum_{j=-M}^{M-1}\left(\exp \left(i(m+n) \pi \frac{j}{M}\right)+\exp \left(-i(m+n) \pi \frac{j}{M}\right)\right)=\left(q_{-}^{-M}+\ldots+q_{-}^{M-1}\right)+ \\
+\left(q_{-}^{M}+\ldots+q_{-}^{1-M}\right)-\left(q_{+}^{-M}+\ldots+q_{+}^{M-1}\right)-\left(q_{+}^{M}+\ldots+q_{+}^{1-M}\right)
\end{gathered}
$$

Each of the four sums in (19) represents a geometric progression.

After summing them, we will have:

$$
\begin{gathered}
\left(q_{-}^{-M}+\ldots+q_{-}^{M-1}\right)+\left(q_{-}^{M}+\ldots+q_{-}^{1-M}\right)-\left(q_{+}^{-M}+\ldots+q_{+}^{M-1}\right)-\left(q_{+}^{M}+\ldots+q_{+}^{1-M}\right)= \\
=q_{-}^{-M}\left(1+\ldots+q_{-}^{2 M-1}\right)+q_{-}^{1-M}\left(1+\ldots+q_{-}^{2 M-1}\right)-q_{+}^{-M}\left(1+\ldots+q_{+}^{2 M-1}\right)- \\
-q_{+}^{1-M}\left(1+\ldots+q_{+}^{2 M-1}\right)=q_{-}^{-M}\left(1+q_{-}\right)\left(1+\ldots+q_{-}^{2 M-1}\right)- \\
-q_{+}^{-M}\left(1+q_{+}\right)\left(1+\ldots+q_{+}^{2 M-1}\right)
\end{gathered}
$$

Let write the auxiliary equality:

$$
\begin{gathered}
S_{-}=\left(1+\ldots+q_{-}^{2 M-1}\right)=1+q_{-}\left(1+\ldots+q_{-}^{2 M-2}\right)= \\
=1+q_{-}\left(1+\ldots+q_{-}^{2 M-2}+q_{-}^{2 M-1}\right)-q_{-}^{2 M}= \\
=1-q_{-}^{2 M}+q_{-} S \Rightarrow S_{-}=\left(1-q_{-}^{2 M}\right) /\left(1-q_{-}\right) \Rightarrow \\
\Rightarrow S_{ \pm}=\left(1+\ldots+q_{ \pm}^{2 M-1}\right)=\left(1-q_{ \pm}^{2 M}\right) /\left(1-q_{ \pm}\right)
\end{gathered}
$$

Using (20) and (21), we get:

$$
\begin{gathered}
4 \sum_{j=-M}^{M-1} \sin \left(n \pi \frac{j}{M}\right) \sin \left(m \pi \frac{j}{M}\right)=\left(q_{-}^{-M}+\ldots+q_{-}^{M-1}\right)+\left(q_{-}^{M}+\ldots+q_{-}^{1-M}\right) \\
-\left(q_{+}^{-M}+\ldots+q_{+}^{M-1}\right)-\left(q_{+}^{M}+\ldots+q_{+}^{1-M}\right)= \\
=q_{-}^{-M}\left(1+q_{-}\right)\left(1-q_{-}^{2 M}\right) /\left(1-q_{-}\right)-q_{+}^{-M}\left(1+q_{+}\right)\left(1-q_{+}^{2 M}\right) /\left(1-q_{+}\right)
\end{gathered}
$$

Since $\left(1-q_{ \pm}\right) \neq 0$ and $\left(1-q_{ \pm}^{2 M}\right)=0$, this implies the proof of equality (16), i.e. the orthogonality of functions $\sin (m \pi j / M)$ on a double segment $[-a, a]$ with a uniform 
partition of the segment with a step $a / M$ into an even number of step.

Let calculate the square of the norm of the functions $\sin (m \pi j / M)$ :

$$
2 \mathbf{N}=2 \sum_{j=-M}^{M} \sin ^{2}\left(m \pi \frac{j}{M}\right)=\sum_{j=-M}^{M}\left(1-\cos \left(2 m \pi \frac{j}{M}\right)\right)
$$

Under the sum sign in (23), let omit the term for $j=0$, which is identically equal to zero, and use the complex Euler formula:

$$
2 \mathbf{N}=\sum_{j=-M, j \neq 0}^{M}\left(1-\frac{1}{2}\left(\exp \left(i 2 m \pi \frac{j}{M}\right)+\exp \left(-i 2 m \pi \frac{j}{M}\right)\right)\right)
$$

Using the notation $q=\exp (i 2 m \pi 1 / M)$, geometric progressions in (24) are summarized as follows:

$$
\begin{gathered}
\sum_{j=-M, j \neq 0}^{M} \exp \left(i 2 m \pi \frac{j}{M}\right)=q^{-M}+\ldots+q^{-1}+q^{1}+\ldots+q^{M}=q^{-M}\left(1+\ldots+q^{M-1}\right)+ \\
+q\left(1+\ldots+q^{M-1}\right)=\left(q+q^{-M}\right)\left(1+\ldots+q^{M-1}\right)=\left(q+q^{-M}\right) \frac{1-q^{M}}{1-q}
\end{gathered}
$$

Since $q^{ \pm M}=\exp ( \pm i 2 m \pi)=1$ and $q \neq 1$, then from (25) we have:

$$
\sum_{j=-M, j \neq 0}^{M} \exp \left(i 2 m \pi \frac{j}{M}\right)=\left(q+q^{-M}\right) \frac{1-q^{M}}{1-q}=0
$$

Then from (24) we obtain $\mathbf{N}=M$ and from (15) we obtain a compact solution to the system:

$$
\psi_{m}=\frac{1}{M} \sum_{j=-M}^{M} \psi\left(x_{j}\right) \sin m \pi \frac{j}{M}, m=-M \div M
$$

For the possibility of applying formula (13), the segment $\left[0, t_{0}\right]$ is divided into $N+1$ equal segments by dots $t=t_{m}=t_{0} m /(N+1), m=0,1,2, . ., N+1 \quad$. Let write equations (8) at each calculation point for $t=t_{m}$ :

$$
\begin{aligned}
& \ddot{x}(0)\left(1-\frac{t_{m}}{t_{0}}\right)+\ddot{x}\left(t_{0}\right) \frac{t_{m}}{t_{0}}-\sum_{m=1}^{N} x_{m}\left(m \pi / t_{0}\right)^{2} \sin m \pi \frac{t_{m}}{t_{0}}+\beta\left[\left(x\left(t_{0}\right)-x(0)\right) / t_{0}+\right. \\
& \left.+\ddot{x}(0)\left(t_{m}-\frac{t_{m}^{2}}{2 t_{0}}-t_{0} / 3\right)+\ddot{x}\left(t_{0}\right)\left(\frac{t_{m}^{2}}{2 t_{0}}-t_{0} / 6\right)+\sum_{m=1}^{N} x_{m} m \pi / t_{0} \cos m \pi \frac{t_{m}}{t_{0}}\right]+ \\
& \quad+\alpha x\left(t_{m}\right) /\left(x^{2}\left(t_{m}\right)+y^{2}\left(t_{m}\right)+z^{2}\left(t_{m}\right)\right)^{3 / 2}=P_{x}\left(t_{m}\right), \\
& \ddot{y}(0)\left(1-\frac{t_{m}}{t_{0}}\right)+\ddot{y}\left(t_{0}\right) \frac{t_{m}}{t_{0}}-\sum_{m=1}^{N} y_{m}\left(m \pi / t_{0}\right)^{2} \sin m \pi \frac{t_{m}}{t_{0}}+\beta\left[\left(y\left(t_{0}\right)-y(0)\right) / t_{0}+\right. \\
& \left.+\ddot{y}(0)\left(t_{m}-\frac{t_{m}^{2}}{2 t_{0}}-t_{0} / 3\right)+\ddot{y}\left(t_{0}\right)\left(-t_{0} / 6\right)+\sum_{m=1}^{N} y_{m} m \pi / t_{0} \cos m \pi \frac{t_{m}}{t_{0}}\right]+ \\
& \quad+\alpha y\left(t_{m}\right) /\left(x^{2}\left(t_{m}\right)+y^{2}\left(t_{m}\right)+z^{2}\left(t_{m}\right)\right)^{3 / 2}=P_{y}\left(t_{m}\right), \\
& \ddot{z}(0)\left(1-\frac{t_{m}}{t_{0}}\right)+\ddot{z}\left(t_{0}\right) \frac{t_{m}}{t_{0}}-\sum_{m=1}^{N} z_{m}\left(m \pi / t_{0}\right)^{2} \sin m \pi \frac{t_{m}}{t_{0}}+\beta\left[\left(z\left(t_{0}\right)-z(0)\right) / t_{0}+\right. \\
& \left.+\ddot{z}(0)\left(t_{m}-\frac{t_{m}^{2}}{2 t_{0}}-t_{0} / 3\right)+\ddot{z}\left(t_{0}\right)\left(\frac{t_{m}^{2}}{2 t_{0}}-t_{0} / 6\right)+\sum_{m=1}^{N} z_{m} m \pi / t_{0} \cos m \pi \frac{t_{m}}{t_{0}}\right]+ \\
& \quad+\alpha z\left(t_{m}\right) /\left(x^{2}\left(t_{m}\right)+y^{2}\left(t_{m}\right)+z^{2}\left(t_{m}\right)\right)^{3 / 2}=P_{z}\left(t_{m}\right) .
\end{aligned}
$$

Let find unknown $x\left(t_{0}\right)$ and $x(0)$ from the boundary conditions (2).

In order to find the unknowns (9), a closed system of nonlinear algebraic equations (28) is obtained.

\section{Finite-Difference Method}

To construct a finite-difference scheme, the time interval $\left[0, t_{0}\right]$ is divided into $n$ equal intervals $\Delta t=\frac{t_{0}}{n}$. Instead of the function of a continuous argument on the segment $\left[0, t_{0}\right]$, let us consider the functions $x\left(t_{i}\right)_{\Delta t}, y\left(t_{i}\right)_{\Delta t}, z\left(t_{i}\right)_{\Delta t}$ of the discrete argument, which are denoted, respectively, by $x_{i}, y_{i}, z_{i}, i=0, . ., n$. The choice of the partition is made in such a way that the ends of the segment participate in it.

The finite-difference approximation of the first derivatives at the $i$-th node is obtained by applying the first-order central difference operator [20]:

$$
\begin{aligned}
& \left.\frac{d x}{d t}\right|_{i}=\frac{x_{i+1}-x_{i-1}}{2 \Delta t}+O\left(\Delta t^{2}\right), \\
& \left.\frac{d y}{d t}\right|_{i}=\frac{y_{i+1}-y_{i-1}}{2 \Delta t}+O\left(\Delta t^{2}\right), \\
& \left.\frac{d z}{d t}\right|_{i}=\frac{z_{i+1}-z_{i-1}}{2 \Delta t}+O\left(\Delta t^{2}\right) .
\end{aligned}
$$

To construct a finite-difference analog of the second derivatives at the $i$-th node, let us use the first-order central difference operator applied twice:

$$
\begin{aligned}
& \left.\frac{d^{2} x(t)}{d t^{2}}\right|_{i}=\frac{x_{i+1}-2 x_{i}+x_{i-1}}{\Delta t^{2}}+O\left(\Delta t^{2}\right), \\
& \left.\frac{d^{2} y(t)}{d t^{2}}\right|_{i}=\frac{y_{i+1}-2 y_{i}+y_{i-1}}{\Delta t^{2}}+O\left(\Delta t^{2}\right), \\
& \left.\frac{d^{2} z(t)}{d t^{2}}\right|_{i}=\frac{z_{i+1}-2 z_{i}+z_{i-1}}{\Delta t^{2}}+O\left(\Delta t^{2}\right) .
\end{aligned}
$$

For such a partition of the segment, the boundary conditions will assume the following form for the functions:

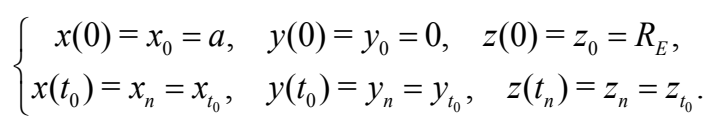

Replacing the derivatives in the original system with their finite-difference analogs (8) and (9), and the known and sought-for functions by their restriction to the grid nodes and discarding the terms of a higher order of smallness, one can arrive at the following system:

$$
\left\{\begin{array}{l}
\frac{x_{i+1}-2 x_{i}+x_{i-1}}{\Delta t^{2}}+\beta \frac{x_{i+1}-x_{i-1}}{2 \Delta t}+\frac{\alpha x_{i}}{\left(x_{i}^{2}+y_{i}^{2}+z_{i}^{2}\right)^{\frac{3}{2}}}=P_{x i}, \\
\frac{y_{i+1}-2 y_{i}+y_{i-1}}{\Delta t^{2}}+\beta \frac{y_{i+1}-y_{i-1}}{2 \Delta t}+\frac{\alpha y_{i}}{\left(x_{i}^{2}+y_{i}^{2}+z_{i}^{2}\right)^{\frac{3}{2}}}=P_{y i}, \\
\frac{z_{i+1}-2 z_{i}+z_{i-1}}{\Delta t^{2}}+\beta \frac{z_{i+1}-z_{i-1}}{2 \Delta t}+\frac{\alpha z_{i}}{\left(x_{i}^{2}+y_{i}^{2}+z_{i}^{2}\right)^{\frac{3}{2}}}=P_{z i} .
\end{array}\right.
$$

Here, $P_{x i}=P_{x}\left(t_{i}\right), P_{y i}=P_{y}\left(t_{i}\right), P_{z i}=P_{z}\left(t_{i}\right), t_{i}=i \Delta t$.

Thus, for each internal node $i, i=1, . ., n-1$, three equations 
with three unknowns are obtained, totally, $3(n-1)$ equations. Adding the boundary conditions (10) to the system, $3(n+1)$ equations with $3(n+1)$ unknowns are obtained, i.e. the system becomes closed. Solving the resulting system, one can find the values of coordinates at fixed time moments $t_{i}=i \Delta t$.

The constructed implicit finite-difference scheme [20] has the second order of accuracy, i.e. with a decrease in the fineness of the partition of the segment $\left[0, t_{0}\right]$, the error decreases proportionally to the square of the grid step $\Delta t$.

\section{Results}

For computational experiments, let us set the values of the parameters included in the system: $t_{0}=30 \mathrm{~s}$, $\omega=\pi / 3600 \mathrm{~s}^{-1}, w=2000 \mathrm{~m} / \mathrm{s}, \quad a=100 \mathrm{~m}, \quad g=9.8 \mathrm{~m} / \mathrm{s}^{2}$, $R_{3}=6372 \mathrm{~km}, \beta=0.1$.

Fig. 1 shows the flight path of the spacecraft calculated using the fast expansion method.

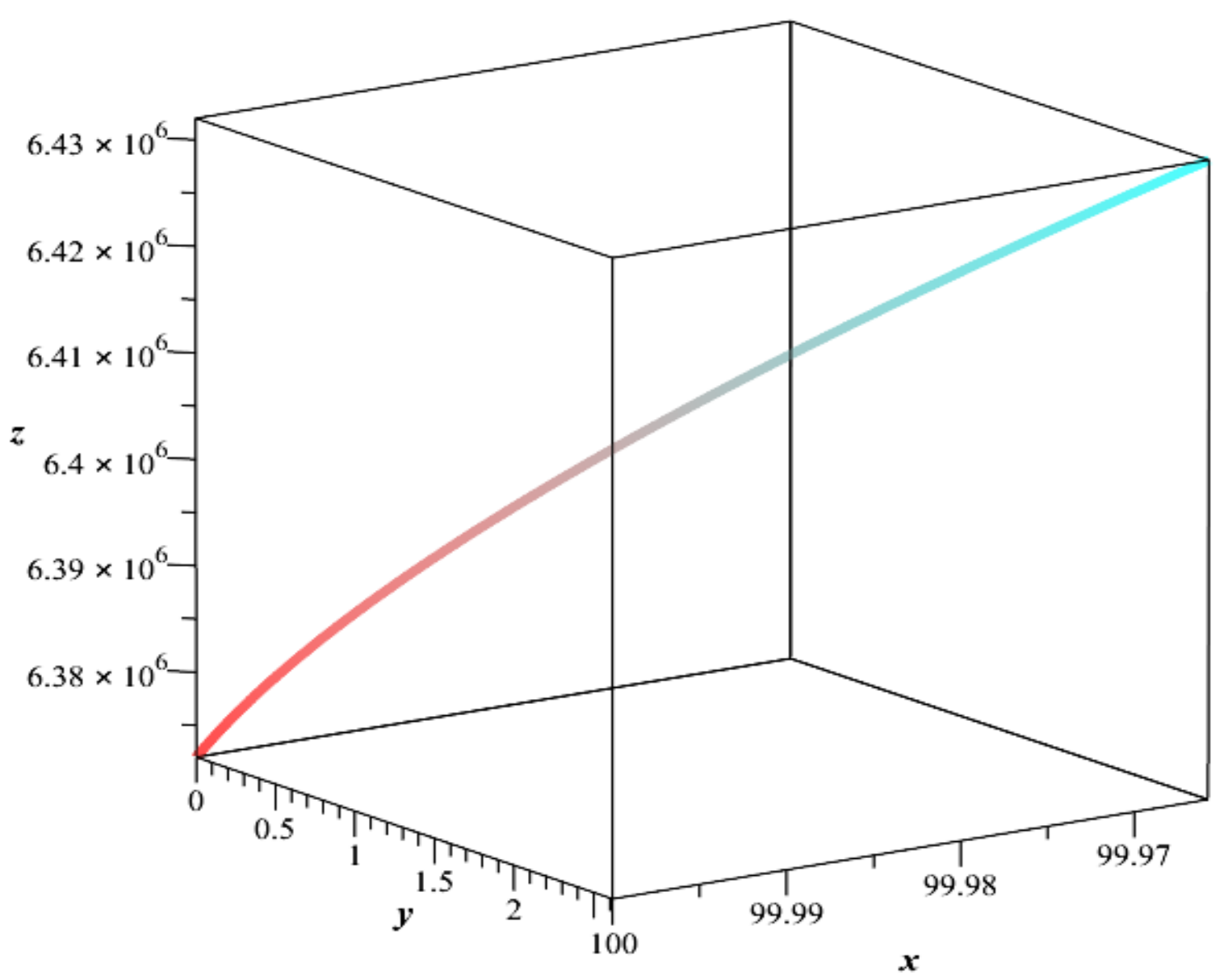

Figure 1. Flight path 
The absolute errors of the spacecraft's trajectory, its velocity and acceleration are calculated using the formulas:

$$
\begin{gathered}
\Delta s=\sqrt{\left(x^{*}-x\right)^{2}+\left(y^{*}-y\right)^{2}+\left(z^{*}-z\right)^{2}}, \\
\Delta v=\sqrt{\left(\dot{x}^{*}-\dot{x}\right)^{2}+\left(\dot{y}^{*}-\dot{y}\right)^{2}+\left(\dot{z}^{*}-\dot{z}\right)^{2}}, \\
\Delta a=\sqrt{\left(\ddot{x}^{*}-\ddot{x}\right)^{2}+\left(\ddot{y}^{*}-\ddot{y}\right)^{2}+\left(\ddot{z}^{*}-\ddot{z}\right)^{2}} .
\end{gathered}
$$

Table 1. Absolute error $\Delta s$ of the trajectory

\begin{tabular}{|c|c|c|c|c|}
\hline $\begin{array}{c}\text { Number } \\
\text { of terms } \\
\text { in the } \\
\text { Fourier } \\
\text { series }\end{array}$ & $M_{2}$ & $M_{4}$ & $M_{6}$ & $M_{8}$ \\
\hline 5 & $2.3 \cdot 10^{-10}$ & $9.2 \cdot 10^{-17}$ & $3.4 \cdot 10^{-23}$ & $1.1 \cdot 10^{-29}$ \\
\hline 10 & $2.2 \cdot 10^{-11}$ & $5.3 \cdot 10^{-18}$ & $1.3 \cdot 10^{-24}$ & $2.7 \cdot 10^{-31}$ \\
\hline 20 & $1.6 \cdot 10^{-12}$ & $1.6 \cdot 10^{-19}$ & $1.7 \cdot 10^{-26}$ & $1.6 \cdot 10^{-33}$ \\
\hline 40 & $1.1 \cdot 10^{-13}$ & $3.4 \cdot 10^{-21}$ & $1.4 \cdot 10^{-28}$ & $5.6 \cdot 10^{-36}$ \\
\hline 80 & $6.8 \cdot 10^{-15}$ & $6.6 \cdot 10^{-23}$ & $8.1 \cdot 10^{-31}$ & $1.2 \cdot 10^{-38}$ \\
\hline
\end{tabular}

Table 1 reflects the change in the error of the solution for various boundary functions and the number of terms in the Fourier series. One can see that, with a twofold increase in the number of terms in the Fourier series, the error decreases by at least one order of magnitude, and with an increase in the order of the boundary function by 2 , the accuracy increases by $6-8$ orders of magnitude.

Table 2. Absolute error $\Delta v$ of the velocity

\begin{tabular}{|c|c|c|c|c|}
\hline $\begin{array}{c}\text { Number } \\
\text { of terms } \\
\text { in the } \\
\text { Fourier } \\
\text { series }\end{array}$ & $M_{2}$ & $M_{4}$ & $M_{6}$ & $M_{8}$ \\
\hline 5 & $1.4 \cdot 10^{-10}$ & $7.2 \cdot 10^{-17}$ & $3.3 \cdot 10^{-23}$ & $1.2 \cdot 10^{-29}$ \\
\hline 10 & $2.3 \cdot 10^{-11}$ & $6.5 \cdot 10^{-18}$ & $1.8 \cdot 10^{-24}$ & $4.3 \cdot 10^{-31}$ \\
\hline 20 & $3.2 \cdot 10^{-12}$ & $3.5 \cdot 10^{-19}$ & $3.9 \cdot 10^{-26}$ & $4.3 \cdot 10^{-33}$ \\
\hline 40 & $4.2 \cdot 10^{-13}$ & $1.4 \cdot 10^{-20}$ & $5.8 \cdot 10^{-28}$ & $2.5 \cdot 10^{-35}$ \\
\hline 80 & $5.5 \cdot 10^{-14}$ & $5.3 \cdot 10^{-22}$ & $6.7 \cdot 10^{-30}$ & $8.9 \cdot 10^{-38}$ \\
\hline
\end{tabular}

Table 3. Absolute error $\Delta a$ of the acceleration

\begin{tabular}{|c|c|c|c|c|}
\hline $\begin{array}{c}\text { Number } \\
\text { of terms } \\
\text { in the } \\
\text { Fourier } \\
\text { series }\end{array}$ & $M_{2}$ & $M_{4}$ & $M_{6}$ & $M_{8}$ \\
\hline 5 & $5.8 \cdot 10^{-11}$ & $3.9 \cdot 10^{-17}$ & $2.2 \cdot 10^{-23}$ & $9.6 \cdot 10^{-30}$ \\
\hline 10 & $1.7 \cdot 10^{-11}$ & $5.6 \cdot 10^{-18}$ & $1.8 \cdot 10^{-24}$ & $4.8 \cdot 10^{-31}$ \\
\hline 20 & $4.6 \cdot 10^{-12}$ & $5.2 \cdot 10^{-19}$ & $6.6 \cdot 10^{-26}$ & $7.7 \cdot 10^{-33}$ \\
\hline 40 & $1.2 \cdot 10^{-12}$ & $4.1 \cdot 10^{-20}$ & $1.8 \cdot 10^{-27}$ & $7.7 \cdot 10^{-35}$ \\
\hline 80 & $3.1 \cdot 10^{-13}$ & $2.9 \cdot 10^{-21}$ & $3.7 \cdot 10^{-29}$ & $5.2 \cdot 10^{-37}$ \\
\hline
\end{tabular}

Tables 2 and 3 present the results of calculations of the absolute error of the velocity and acceleration of the spacecraft. The absolute velocity error decreases with the slightly lower velocity, and the acceleration is even slower compared to the trajectory error with an increase in the number of members of the series of fast sine expansion. However, with an increase in the order of the boundary function, all three errors behave identically.

Table 4. Calculation time (s)

\begin{tabular}{|c|c|c|c|c|}
\hline $\begin{array}{c}\text { Number of } \\
\text { terms in the } \\
\text { Fourier series }\end{array}$ & $M_{2}$ & $M_{4}$ & $M_{6}$ & $M_{8}$ \\
\hline 5 & 2 & 3 & 4 & 5 \\
\hline 10 & 3 & 4 & 6 & 8 \\
\hline 20 & 9 & 11 & 14 & 20 \\
\hline 40 & 50 & 55 & 72 & 81 \\
\hline 80 & 330 & 408 & 482 & 1,512 \\
\hline
\end{tabular}

Table 5. The minimum number of significant digits in the calculation

\begin{tabular}{|c|c|c|c|c|}
\hline $\begin{array}{c}\text { Number of } \\
\text { terms in the } \\
\text { Fourier } \\
\text { series }\end{array}$ & $M_{2}$ & $M_{4}$ & $M_{6}$ & $M_{8}$ \\
\hline 5 & 15 & 22 & 29 & 35 \\
\hline 10 & 20 & 24 & 30 & 37 \\
\hline 20 & 25 & 25 & 35 & 40 \\
\hline 40 & 40 & 35 & 40 & 45 \\
\hline 80 & 60 & 70 & 75 & 80 \\
\hline
\end{tabular}

Tables 4 and 5 contain information about the complexity of the computational experiment, i.e. the calculation time and the number of significant digits. As seen from Table 4, the calculation time increases sharply with an increase in the number of terms in the Fourier series, whereas an increase in the order of the boundary function leads to an insignificant increase in the calculation time. Having analyzed the data in Tables $1-5$, one concludes that, in order to achieve high accuracy of the solution, it is necessary to increase the order of the boundary function (3), and leave the number of terms in the Fourier series minimal.

Table 6 presents the data of errors of the trajectory, velocity and acceleration obtained using the classical method of finite differences. The smallest error is of the order of $10^{-9}$, which corresponds to the fast expansion method with the boundary function $M_{2}$ and 5 terms in the Fourier series. However, the calculation time of the finite-difference method exceeds by more than 650 times the corresponding time of the fast expansion method. 
Table 6. Errors of the finite difference method

\begin{tabular}{|c|c|c|c|c|}
\hline \multirow{2}{*}{ Time step $\Delta t, \mathbf{s}$} & \multicolumn{3}{|c|}{ Absolute errors } & Calculation \\
\cline { 2 - 5 } & $\Delta s$ & $\Delta v$ & $\Delta a$ & \multirow{2}{*}{ Significant digits } \\
\hline 1 & $1.1 \cdot 10^{-7}$ & $3.2 \cdot 10^{-8}$ & $3.2 \cdot 10^{-9}$ & 3 \\
\hline $1 / 2$ & $2.8 \cdot 10^{-8}$ & $8.3 \cdot 10^{-9}$ & $8.3 \cdot 10^{-10}$ & 21 \\
\hline $1 / 4$ & $6.8 \cdot 10^{-9}$ & $2.6 \cdot 10^{-9}$ & $2.6 \cdot 10^{-10}$ & 17 \\
\hline $1 / 8$ & $1.7 \cdot 10^{-9}$ & $5.3 \cdot 10^{-10}$ & $5.3 \cdot 10^{-11}$ & 20 \\
\hline
\end{tabular}

Table 7. Errors in the powered portion of the flight

\begin{tabular}{|c|c|c|c|c|}
\hline \multirow{2}{*}{$\begin{array}{c}\text { Number of terms } \\
\text { in the Fourier } \\
\text { series }\end{array}$} & $\Delta s$ & $\Delta v$ & \multicolumn{2}{|c|}{ Absolute errors } \\
\cline { 2 - 5 } & $9.1 \cdot 10^{-17}$ & $7.1 \cdot 10^{-17}$ & $3.2 \cdot 10^{-17}$ \\
\hline 5 & $5.3 \cdot 10^{-18}$ & $6.2 \cdot 10^{-18}$ & $5.5 \cdot 10^{-18}$ \\
\hline 10 & $1.5 \cdot 10^{-19}$ & $3.6 \cdot 10^{-19}$ & $5.2 \cdot 10^{-19}$ \\
\hline 20 & $3.5 \cdot 10^{-21}$ & $1.4 \cdot 10^{-20}$ & $4.1 \cdot 10^{-20}$ \\
\hline 40 & $6.1 \cdot 10^{-23}$ & $5.1 \cdot 10^{-22}$ & $2.9 \cdot 10^{-21}$ \\
\hline 80 & & & 60 \\
\hline
\end{tabular}

\section{Powered Portion of the Flight}

The motion of a spaceship without the mass loss is possible only with the engine turned off, i.e. on the unpowered portion of the flight. Let us generalize the formulation of the problem for the powered portion of the trajectory. Suppose that fuel is consumed according to a linear law, therefore, the mass of the spaceship is expressed by the function $m(t)=m_{0}(1-\lambda t)$, where $m_{0}$ is the starting mass, $\lambda$ is the proportionality factor. In this case, the equations of motion will assume the form:

$$
\left\{\begin{array}{l}
m_{0}(1-\lambda t) \ddot{x}+\beta \dot{x}+\alpha m_{0}(1-\lambda t) x /\left(x^{2}+y^{2}+z^{2}\right)^{3 / 2}=P_{x}, \\
m_{0}(1-\lambda t) \ddot{y}+\beta \dot{y}+\alpha m_{0}(1-\lambda t) y /\left(x^{2}+y^{2}+z^{2}\right)^{3 / 2}=P_{y}, \\
m_{0}(1-\lambda t) \ddot{z}+\beta \dot{z}+\alpha m_{0}(1-\lambda t) z /\left(x^{2}+y^{2}+z^{2}\right)^{3 / 2}=P_{z} .
\end{array}\right.
$$

Let us divide each equation by $m_{0}$ and denote $\beta_{0}=\frac{\beta}{m_{0}}$ and $P_{x 0}=\frac{P_{x}}{m_{0}}, P_{y 0}=\frac{P_{y}}{m_{0}}, P_{z 0}=\frac{P_{z}}{m_{0}}$; finally one gets:

$$
\left\{\begin{array}{c}
(1-\lambda t) \ddot{x}+\beta_{0} \dot{x}+\alpha(1-\lambda t) x /\left(x^{2}+y^{2}+z^{2}\right)^{3 / 2}=P_{x 0}, \\
(1-\lambda t) \ddot{y}+\beta_{0} \dot{y}+\alpha(1-\lambda t) y /\left(x^{2}+y^{2}+z^{2}\right)^{3 / 2}=P_{y 0}, \\
(1-\lambda t) \ddot{z}+\beta_{0} \dot{z}+\alpha(1-\lambda t) z /\left(x^{2}+y^{2}+z^{2}\right)^{3 / 2}=P_{z 0} .
\end{array}\right.
$$

For numerical calculation, let us set $\lambda=\frac{1}{2 t_{0}}, \beta_{0}=0.1$, the other parameters included in both the equation and the test function will be left unchanged. The components of the reactive force are selected so that there is the same exact solution to the problem.

Table 7 shows the absolute errors of the calculation by the fast expansion method with the boundary function $M_{4}$.
An analysis of the table data shows the same dynamics of change in error as in the case with constant mass, and with the same order of accuracy for the boundary function $M_{4}$. With a twofold increase in the number of terms in the Fourier series, the error decreases by at least one order of magnitude. The velocity and acceleration errors are of the same order as the error of trajectory for 5,10 , and 20 members of the Fourier series, and differ by an order of magnitude for 80 members.

Thus, the fast expansion method demonstrates excellent results also for calculating the trajectory in the powered portion of the spacecraft flight.

\section{Conclusions}

Using the fast expansion method, an approximate analytical solution to the problem of the motion of a spacecraft from a starting point to a final point during a certain time has been found. The study of the obtained solution has revealed that, in order to achieve high accuracy of the solution in the fast expansion method, it is necessary to increase the order of the boundary function, and leave the number of terms in the Fourier series minimal. The original problem was also solved by the classical finite-difference method using a finite-difference scheme of the second order of accuracy. A comparative analysis showed that, with the same calculation error, the calculation time of the fast expansion method was many hundred times smaller. Moreover, because of limited computer resources, it is impossible to achieve an accuracy of the order of $10^{-38}$, obtained by the fast expansion method, using finite differences. Another advantage of the fast expansion method is that the solution is obtained in an analytical form. The fast expansion method is very 
effective in solving boundary value problems for ordinary differential equations and their systems.

\section{REFERENCES}

[1] D. Chernyshov, V. V. Goryainov, O. A. Chernyshov. Application of the fast expansion method for spacecraft trajectory calculation, Russian Aeronautics, Vol. 58, No 2, 180-186, 2015. doi.org/10.3103/S1068799815020075.2.

[2] L. P. Mukhamedov, D. A. Kirievsky. Priblizhennaya metodika proyektirovochnogo ballisticheskogo rascheta pervykh stupeney raket-nositeley [Approximate method of design ballistic calculation of the first stages of launch vehicles], Izvestiya vysshikh uchebnykh zavedeniy. Mashinostroyeniye, Vol. 6, 67-77, 2020. doi.org/10.18698/0536-1044-2020-6-67-773.

[3] R. Chai, A. Savvaris, A. Tsourdos, S. Chai, Y. Xia. A review of optimization techniques in spacecraft flight trajectory design. Progress in Aerospace Sciences, Vol. 109, 100543, 2019. doi.org/10.1016/j.paerosci.2019.05.003.4.

[4] N. L. Sokolov. Analiticheskiy metod issledovaniya optimalnogo upravleniya KA pri dvizhenii $\mathrm{v}$ atmosfere [Analytical method for studying the optimal control of a spacecraft during movement in the atmosphere], Lesnoy vestnik, Vol. 19, No. 3, 37-44, 2015.

[5] N. L. Sokolov. Analiticheskii metod rascheta traektorii dvizheniya kosmicheskogo apparata pri provedenii raketodinamicheskikh manevrov i korrektsii [The Analytical Method for Calculating the Trajectories of a Spacecraft during Rocket-Dynamic Maneuvers and Corrections], Informatsiya i kosmos, Vol. 2, 127-133, 2016.

[6] V. V. Karagodin. The approximate calculation methods of the extra-atmospheric active trajectory leg, Trudy MAI, Vol. $66,1-12,2013$.

[7] Gong, S. Li, J. Shi, Y. Yang. Rotation based analytic range-only initial relative orbit solution for natural periodic motion, Acta Astronautica, Vol. 178, 584-594, 2021. doi.org/10.1016/j.actaastro.2020.09.050

[8] S. V. Benevolskii, P. G. Kozlov. Poluanaliticheskii metod vosstanovleniya traektorii LA po obobshchennym proektnym parametram i parametram programy upravleniya i perspektivy ego ispolzovaniya [A Semi-Analytical Method for Reconstructing Aircraft Trajectories from Generalized Design Parameters and Control Program Parameters and Prospects for Its Use], Nauka i obrazovanie, Vol. 13, 2011.

[9] V. G. Kozlov, V. A. Gulevsky, A. V. Skrypnikov, V. S. Logoyda, A. S. Menzhulova. Method of Individual Forecasting of Technical State of Logging Machines, IOP Conference Series: Materials Science and Engineering, Vol. 327, No. 4, 042056, 2018.

[10] V. G. Kozlov, A. V. Skrypnikov, E. V. Chernyshova, R. V. Mogutnov, D. M. Levushkin. Mathematical modeling of damage function when attacking file server, Journal of Physics: Conference Series, Vol. 1015, No. 3, 032069 , 2018.
[11] H. Naher, H. Shafia, Md. E. Ali, G. C. Paul. A Comparative Study of Space and Time Fractional KdV Equation through Analytical Approach with Nonlinear Auxiliary Equation, Mathematics and Statistics, Vol. 8, No. 1, 1-16, 2020. doi.org/10.13189/ms.2020.080101

[12] S. Yena, N. Pochai. Numerical Simulation of a Two-Dimensional Vertically Averaged Groundwater Quality Assessment in Homogeneous Aquifer Using Explicit Finite Difference Techniques, Mathematics and Statistics, Vol. 8, No. 2, 152-165, 2020. doi.org/10.13189/ms.2020.080211

[13] G. Kozlov, P. V. Tikhomirov, V. Y. Bukreev, M. A. Abasov, V. A. Khvostov. Accelerated convergence of numerical solution to square plate bending problem, ARPN Journal of Engineering and Applied Sciences, Vol. 19, No 1, 969-976, 2020

[14] S. O. Forbes-Spyratos, M. P. Kearney, M. K. Smart, I. H. Jahn. Trajectory Design of a Rocket-Scramjet-Rocket Multistage Launch System, Journal of Spacecraft and Rockets, Vol. 56, 1-14, 2018. doi.org/10.2514/1.A34107

[15] D. Chernyshov, M. I. Popov, O. Yu Nikiforova, V. V. Goryainov. Using of fast expansions in the construction of two-dimensional exact solutions of the Poisson equation, Journal of Physics: Conference Series, Voronezh, November 11-13, 2019, IOP Publishing Ltd, Voronezh, 2020. doi.org/10.1088/1742-6596/1479/1/012146.

[16] V. V. Goryainov, M. I. Popov, A. D. Chernyshov. Solving the Stress Problem in a Sharp Wedge-Whaped Cutting Tool Using the Quick Decomposition Method and the Problem of Matching Boundary Conditions, Mechanics of Solids, Vol. 54, No 7, 1083-1097, 2019. doi.org/10.3103/S002565 4419070094 .

[17] D. Chernyshov, M. I. Popov, D. A. Litvinov. High accurate sine and cosine interpolation with repeated differentiation ability obtained by fast expansions technique, Journal of Physics: Conference Series: Current Problems, Voronezh, December 07-09, 2020, Voronezh, 2021. doi.org/10.1088/ 1742-6596/1902/1/012026.18.

[18] D. Chernyshov, V. V. Goryainov, O. A. Chernyshov. Raschet poleta kosmicheskogo korablya na vneatmosfernom uchastke trayektorii metodom bystrykh razlozheniy [Calculation of the flight of a spacecraft in the extra-atmospheric section of the trajectory by the fast expansion method], Theoretical \& Applied Science, Vol. 6, 2014, 1-4.

[19] D. Chernyshov, V. V. Goryainov. O svyazi kolichestva tochek peregiba grafika funktsii i kolichestva chlenov ryadov Furye pri ispolzovanii bystrykh razlozheniy [On the relationship between the number of inflection points of a function graph and the number of members of Fourier series when using fast expansions], ISJ Theoretical \& Applied Science, Vol. 1, No. 33, 137-141, 2016.

[20] M. I. Popov. Integrirovaniye bigarmonicheskogo uravneniya po neyavnoy skheme [Integration of the biharmonic equation according to an implicit scheme], Bulletin of the Voronezh State University of Engineering Technologies, Vol. 80, No. 2(76), 114-118, 2018. 